\title{
Cell Type-Specific Gene Expression of Alpha 5 Subunit-Containing Gamma-Aminobutyric Acid Subtype A Receptors in Human and Mouse Frontal Cortex
}

\author{
Xiyue Hua,b Brad R. Rocco ${ }^{a}$ Corey Fee ${ }^{a, b}$ Etienne Sibille ${ }^{a-c}$ \\ ${ }^{a}$ Campbell Family Mental Health Research Institute of CAMH, Toronto, ON, Canada; bepartment of Pharmacology \\ and Toxicology, University of Toronto, Toronto, ON, Canada; ' Department of Psychiatry, University of Toronto, \\ Toronto, ON, Canada
}

\section{Keywords}

Gamma-aminobutyric acid · GABRA5 · Cortical

microcircuitry · Somatostatin · Fluorescence in situ

hybridization

\begin{abstract}
Converging evidence suggests that deficits in somatostatin (SST)-expressing neuron signaling contributes to major depressive disorder. Preclinical studies show that enhancing this signaling, specifically at a5 subunit-containing $\gamma$-aminobutyric acid subtype $A$ receptors (a5-GABA $A_{A} R s$ ), provides a potential means to overcome low SST neuron function. The cortical microcircuit comprises multiple subtypes of inhibitory $\gamma$-aminobutyric acid (GABA) neurons and excitatory pyramidal cells (PYCs). In this study, multilabel fluorescence in situ hybridization was used to characterize a5-GABA $R$ gene expression in PYCs and three GABAergic neuron subgroups - vasoactive intestinal peptide (VIP)-, SST-, and parvalbumin (PV)-expressing cells - in the human and mouse frontal cortex. Across species, we found the majority of gene expression in PYCs (human: 39.7\%; mouse: 54.14\%), less abundant expression in PV neurons (human: 20\%; mouse: $16.33 \%)$, and no expression in VIP neurons (0\%). Only human
\end{abstract}

\section{KARGER}

(c) 2019 S. Karger AG, Basel

E-Mail karger@karger.com

www.karger.com/mnp
SST cells expressed GABRA5, albeit at low levels (human: 8.3\%; mouse: $0 \%$ ). Together, this localization suggests potential roles for a5-GABA $A_{A}$ Rs within the cortical microcircuit: (1) regulators of PYCs, (2) regulators of PV cell activity across species, and (3) sparse regulators of SST cell inhibition in humans. These results will advance our ability to predict the effects of pharmacological agents targeting a5-GABA ${ }_{A} R s$, which have shown therapeutic potential in preclinical animal models.

(c) 2019 S. Karger AG, Basel

\section{Introduction}

Multiple brain functions, from sensory processing to cognitive computations, originate from the propagation of excitatory signals across interconnected layers, regions, and networks of the neocortex. These signals are transmitted by glutamatergic pyramidal cells (PYCs) and shaped through tight regulation by diverse subgroups of local interneurons that express the inhibitory neurotransmitter $\gamma$-aminobutyric acid (GABA) [1]. Within these assembled microcircuits, excitatory activity is balanced by dynamic inhibition at several levels to produce coherent 
neuronal output necessary for normal functions. However, in disease, the balance between excitation and inhibition can become dysregulated, potentially supporting the emergence of psychiatric symptoms rooted in altered perception, cognition, mood, and even motor functions. Indeed, robust evidence of reductions in GABA levels together with deficits in cortical inhibitory function among subjects with major depressive disorder (MDD) suggests that altered excitation-inhibition balance plays a central role in MDD etiology [2].

GABAergic neuron populations represent a minority of all cortical neurons (rodents: 15\%; humans: 25\%) [36]; however, their innervations onto PYCs are essential to maintain the excitation-inhibition balance of the entire cortical network. A large diversity of GABAergic neurons can be classified based on electrophysiological, morphological, and neurochemical properties [7]. In rodents, the vast majority of cortical interneurons $(\sim 100 \%)$ can be divided into three nonoverlapping groups based on coexpression of distinct neurochemical markers, namely that of the neuropeptide somatostatin (SST; 30\%), the calcium-binding protein parvalbumin (PV; 40\%), and the ionotropic serotonin receptor 5HT3aR (30\%), which largely comprises vasoactive intestinal peptide (VIP)expressing neurons [8]. In humans, the majority of GABAergic neurons $(\sim 85 \%)$ in the prefrontal cortex (PFC) can be differentiated based on nonoverlapping expression of the calcium-binding proteins PV (20\%), calbindin $(20 \%)$, or calretinin $(45 \%)[5,9,10]$.

Evidence from postmortem MDD studies shows that SST cells are selectively vulnerable among GABAergic deficits across the dorsolateral PFC [11-13], amygdala [14], and subgenual anterior cingulate cortex [15]. SST cell inhibition primarily targets PYC dendrites, gating the input of excitatory signals from thalamocortical afferents or upstream microcircuitry, a function that is partially mediated by activation of dendritic $\alpha 5$ subunit-containing GABA subtype A receptors ( $\left.\alpha 5-\mathrm{GABA}_{\mathrm{A}} \mathrm{Rs}\right)[16,17]$. Thus, enhancing (or modulating) signaling specifically at a5-GABA ${ }_{A}$ Rs provides a potential means to restore, or overcome, the low SST cell function found in MDD [18]. Indeed, a growing number of experimental manipulations in rodents suggest that remediating low SST cell function may lead to antidepressant-like effects (see review in Fee et al. [19]).

Although the impact of enhancing GABAergic input via modulation of PYC a5-GABA ${ }_{A}$ Rs seems somewhat straightforward, GABAergic neurons also express $\mathrm{GABA}_{\mathrm{A}}$ Rs [20]. Different GABAergic cell types vary by their targeted cellular compartments, the cell types they

Cell Type-Specific Gene Expression of a5-GABA $\mathrm{A}$ Rs interact with, and the receptors that mediate their effects. For instance, PV cells mainly target the soma and proximal dendrites of PYCs with synapses that are enriched with a1-GABA $A_{\mathrm{A}}$ Rs [21]. Regarding interactions between GABAergic neurons, PV-PV reciprocal connections are predominant, but these cells exert only weak inhibition on other GABAergic neuron subtypes [22]. In contrast, SST cells strongly inhibit PYC dendrites and select GABAergic (e.g., PV, VIP) neurons, but do not target each other [22]. VIP cells play a central role in PYC disinhibition by preferentially targeting SST neurons [23, $24]$. These diverse properties dramatically increase the complexity of the cortical microcircuit. Therefore, understanding how the activity of the cortical microcircuit may be modulated by pharmacological targeting of a5$\mathrm{GABA}_{\mathrm{A}}$ Rs is important to advance our ability to develop effective treatments through this candidate mechanism.

Here, we used multilabel fluorescence in situ hybridization (FISH) to characterize the cell type distributions of PFC a5-GABA $\mathrm{A}_{\mathrm{A}} \mathrm{Rs}$ by investigating gene expression patterns (mouse: Gabra5; human: GABRA5) in glutamatergic PYCs via SLC17A7, a marker for the vesicular glutamate transporter 1 , and in three major groups of GABAergic neurons via their neurochemical markers: PV, SST, and VIP. To assess the viability of translating a5-selective compounds to clinical use, we performed multiple analyses to characterize the cell type-specific expression pattern of GABRA5/Gabra5 between mice and humans. We predicted that GABRA5/Gabra5 expression patterns would be similar between mouse and human PFC. We further predicted that PYCs would have high GABRA5/Gabra5 expression due to established roles for SST cells in dendritic inhibition. As mentioned earlier, PV- and VIP-expressing cells are also targeted by SST cells. Based on this, we also predicted that small proportions of these GABAergic cell types would express GABRA5/Gabra5. Finally, since SST cells do not share reciprocal connections, we predicted that these cells would have limited or no GABRA5/Gabra5 expression.

\section{Materials and Methods}

Mouse and Human Brain Samples

Fresh brains from adult wild-type C57BL/ 6 mice $(n=6 ; 50 \%$ male; 4 months old) were extracted following sacrifice via cervical dislocation. Brains were immediately frozen with dry ice and stored at $-80^{\circ} \mathrm{C}$ until processing. The Douglas-Bell Canada Brain Bank (Douglas Mental Health University Institute, Montreal, QC, Canada) provided human postmortem tissue blocks that contained the PFC from 5 male human subjects with no known psy- 
chiatric or neurological disorder (online suppl. Table 1; for all online suppl. material, see www.karger.com/doi/10.1159/000495840). The subjects selected were matched as closely as possible for age, postmortem interval, and brain $\mathrm{pH}$. Coronal PFC sections with a thickness of 14 and $12 \mu \mathrm{m}$ were prepared via cryostat for mice and humans, respectively. Tissue sections were mounted onto glass slides and stored at $-80^{\circ} \mathrm{C}$ until processed for FISH.

\section{Fluorescence in situ Hybridization}

RNAscope FISH technology was used in this study. FISH probes were designed by Advanced Cell Diagnostics (Newark, CA, USA) to label mouse tissues for mRNA encoding Gabra5 (product \#319481), Pv (product \#421931), Sst (product \#404631), Slc17a7 (product \#416631), Vip (product \#415961), and human tissues for mRNA encoding GABRA5 (product \#452741), PV (product \#422181), SST (product \#310591), SLC17A7 (product \#415611), and VIP (product \#452751). Two separate experiments were performed, each using one section from all subjects $(n=6)$, to label Gabra5 in (1) Slc17a7- and Vip-expressing cells or in (2) $P v$ - and $S s t$-expressing cells. The labeling procedure was performed according to the manufacturer's protocol [25], with a few exceptions, and was performed the same for both mouse and human tissue. After fixation in 4\% paraformaldehyde for $1 \mathrm{~h}$, sections were serially dipped in 50, 70, and 100\% ethanol diluted with water, each for $5 \mathrm{~min}$. Sections were treated with protease III for $30 \mathrm{~min}$ at room temperature, rinsed with phosphate-buffered saline, and incubated with probe solution for $2 \mathrm{~h}$ at $40^{\circ} \mathrm{C}$ using an oven designed with HybEZ Hybridization System (Advanced Cell Diagnostics). A series of fluorescent multiplex detection reagents (AMP 1-4) were used to amplify the hybridization signals of label probes on target RNA. Sections were washed twice with wash buffer after each AMP treatment. Slc17a7 and $P v$ mRNAs were detected with Atto 550, Vip and Sst mRNAs were detected with Atto 647, and Gabra5 mRNA was detected with Alexa Fluor ${ }^{\circledR} 488$. The same approach was used for human analyses $(n=5)$, except that GABRA5 mRNA was detected with Atto 550, VIP and $P V$ were detected with Atto 647, and SLC17A7 and SST were detected with Alexa Fluor ${ }^{\circledR} 488$. After the final wash, sections were counterstained with NeuroTrace blue fluorescent Nissl stain (product \#N21479; Invitrogen) diluted to 1:100 and 1:50 in phosphatebuffered saline for mice and humans, respectively, for $20 \mathrm{~min}$ at room temperature. The sections were then rinsed in phosphatebuffered saline and ProLong Gold Antifade Mountant (product \#P36930; Invitrogen) was applied directly to the fluorescent-labeled tissue and sealed with a coverslip. All sections were stored at $4^{\circ} \mathrm{C}$ until imaging.

\section{Wide-Field Fluorescence Microscopy}

Images were acquired with an Olympus IX83 microscope (Richmond Hill, ON, Canada) equipped with a Hamamatsu ORCA-Flash4.0 V2 digital CMOS camera (Bridgewater, NJ, USA) and high-precision ProScan III XYZ motorized stage with linear XYZ encoders (Prior Scientific, Rockland, MA, USA). The hardware was controlled by SlideBook6 (Intelligent Imaging Innovations, Inc., Denver, CO, USA), which was also the software used for image processing. Both mouse and human data were collected using a $60 \times 1.40$ NA super corrected oil immersion objective. For each brain section, 10 randomly sampled $3 \mathrm{D}$ image stacks (2D images captured using a step size separated by $0.25 \mu \mathrm{m}$ in the $\mathrm{z}$-dimension) were collected in the superficial layer based on sampling of layer II/III, and 10 were collected in the deep layer based on sampling of layer IV/V. The stacks were $1,024 \times 1,024$ pixels $(\sim 111$ $\times 111 \mu \mathrm{m})$. All images were acquired over the entire thickness of the tissue section using optimal exposure settings. For the imaging of the human sections, lipofuscin autofluorescence was detected as described [26].

\section{Image Processing}

Each fluorescent channel was deconvolved using the AutoQuant adaptive blind deconvolution algorithm (Media Cybernetics, Inc., Rockville, MD, USA). All NeuroTrace-labeled cells whose center, as defined by the largest surface area, was contained within an $82.9 \times 82.9 \mu \mathrm{m}^{2}$ counting frame placed in the center of each stack and beginning and ending one $z$-plane above and below the first and last z-plane of the stack, respectively, were manually traced. The mask was then copied to the adjacent top and bottom z-plane. Data segmentation was performed in MATLAB (R2016) on the channels used to detect mRNAs and lipofuscin, as previously described [26]. Briefly, a new channel was created by calculating a difference of 3D Gaussian filtered channels using sigma values of 0.7 and 2. The Ridler-Calvard automated thresholding algorithm was used to calculate an initial value for iterative segmentation with subsequent iterations increasing by 25 gray scale values. The segmented object masks were size-gated between 0.03 and $0.07 \mu \mathrm{m}^{3}$ for mice and humans. All mRNA object masks that overlapped with a NeuroTrace-labeled cell mask were assigned to that cell. For the human images, any mRNA grain masks that overlapped a lipofuscin mask were removed from analysis. Each image was manually screened for masking accuracy.

\section{Classification of Cells}

Among the cells that were manually traced as described earlier, cells containing $\geq 10 \mathrm{mRNA}$ grains were considered to be positive cell marker-expressing cells. This cutoff was based on the frequency distribution graph (online suppl. Fig. 1).

\section{Statistics}

All mRNA objects that overlapped with a cell mask were included in the analysis. The GABRA5/Gabra5 mRNA expression level was quantified by a descriptive analysis of grain counting.

\section{Results}

\section{Gabra5 Expression in Mouse PFC}

A total of 2,812 cells were included in the study. Within mouse PFC tissue sections we identified 1,027 Slc17a7expressing cells, 42 Vip-expressing cells, 51 Sst-expressing cells, and $49 P v$-expressing cells. We then quantified cells labeled for Slc17a7 (Fig. 1a), Vip (Fig. 1b), Sst (Fig. 1c), and Pv (Fig. 1d) with and without Gabra5 colocalized. Cell types were first identified, with $\geq 10$ mRNA grains per cell body defining positive expression (Fig. 2a). Cell density per $100 \mu \mathrm{m}^{3}$ was then quantified (Fig. 2b). The proportion of Slc17a7-expressing cells and GABAergic neurons (VIP, SST, and PV) was 87.9 and $12.1 \%$, respectively. The ratios of sampled GABAergic neurons ex- 


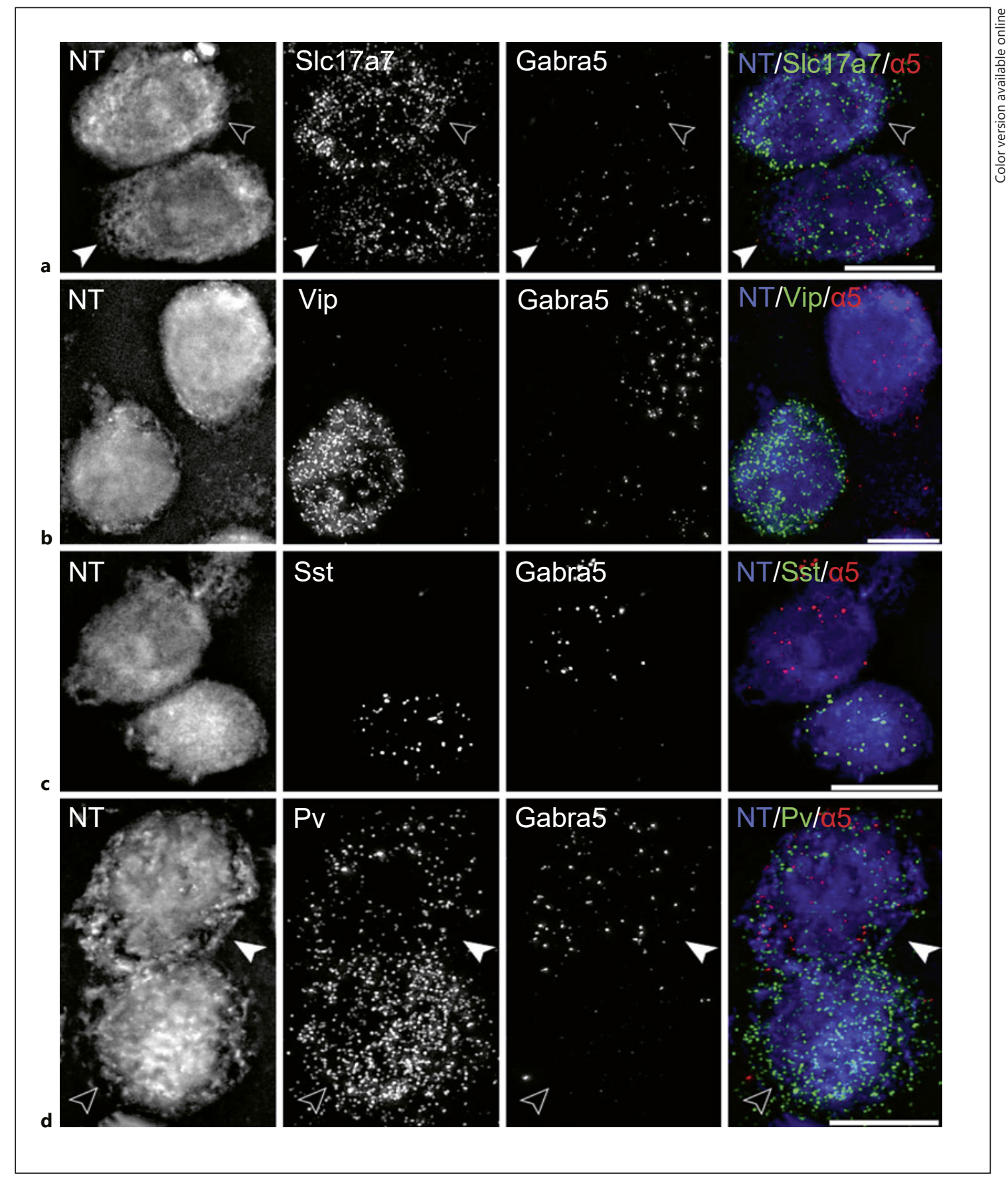

Fig. 1. Gabra5 expression in Slc17a7-, Vip-, Sst-, and Pv-expressing cells in mouse PFC. Projection images (three z-planes separated by $0.25 \mu \mathrm{m}$ ) of a mouse PFC tissue section, counterstained with NeuroTrace blue, and labeled for Slc17a7 with and without Gabra5 (a), Vip without Gabra5 (b), Sst without Gabra5 (c), and Pv with and without Gabra5 (d). Solid arrowheads depict Gabra5-expressing neurons. Scale bars, $10 \mu \mathrm{m}$.

pressing Vip, Sst, and $P v$ were 29.6, 35.9, and 34.5\%, respectively. For cells identified as one of these neuronal subtypes, a scatter plot was generated to quantify Gabra5 mRNA grains for each cell type (Fig. 2c). In mice, the per- centages of Slc17a7-, Vip-, Sst-, and Pv-expressing cells that also expressed Gabra5 were 54.14, 0, 0, and $16.33 \%$, respectively. 


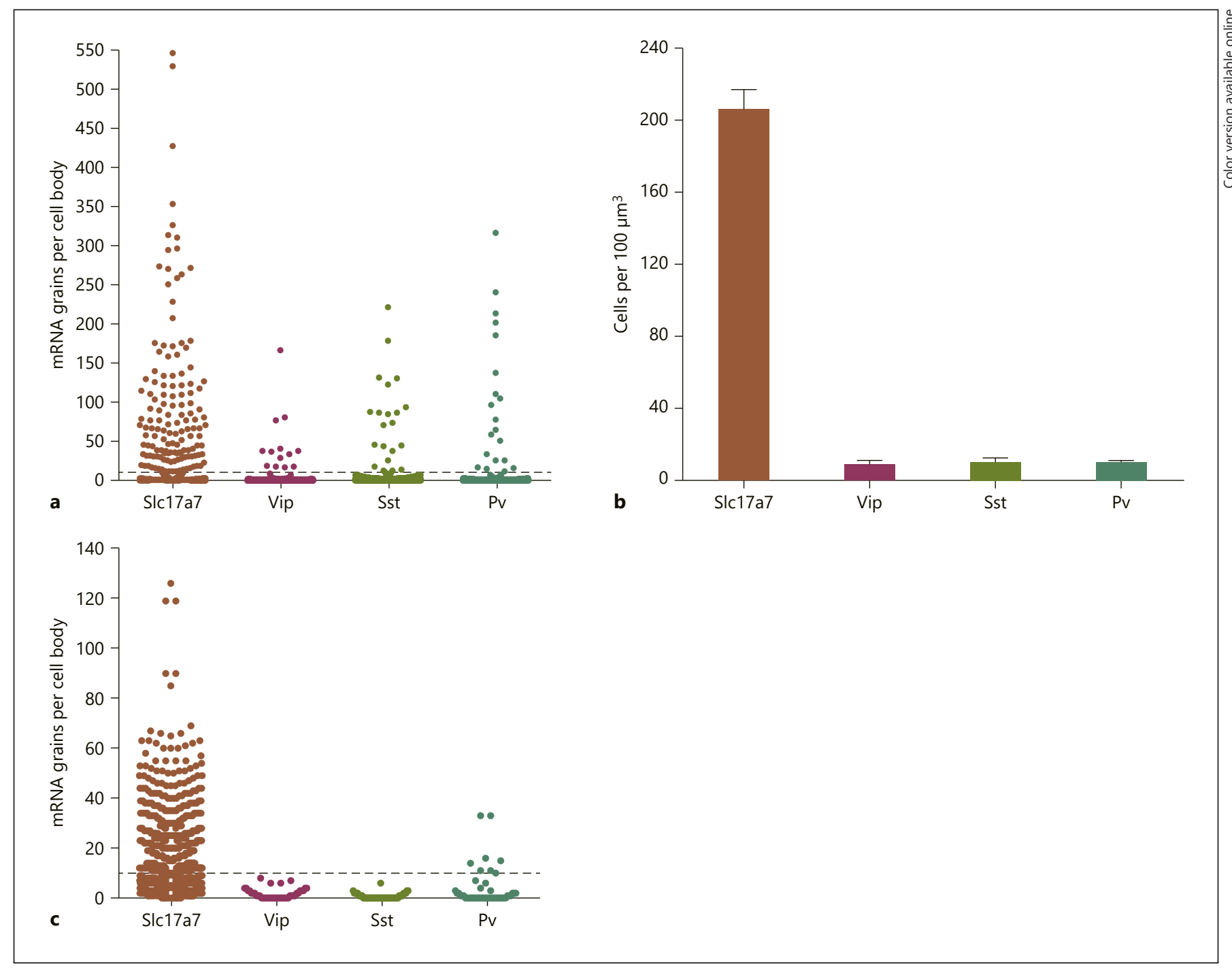

Fig. 2. Quantification of Gabra5 mRNA grains per cell body across cell types in mouse PFC. Cells with $\geq 10$ mRNA grains are defined as positive cell marker-expressing cells. a Cell marker mRNA grains per cell body distinguish different cell types. b Cell density of different cell types per $100 \mu \mathrm{m}^{3}$ mouse PFC. c Gabra5 mRNA grains per cell body expressed in Slc17a7-, Vip-, Sst-, or Pv-expressing cells.

\section{GABRA5 Expression in Human PFC}

The same approach was used in the human postmortem cohort to quantify GABRA5 mRNA expression in SLC17A7-, VIP-, SST-, and PV-expressing cells. A total of 1,089 cells were included in the study. We identified 146 SLC17A7-expressing cells, 13 VIP-expressing cells, 24 SST-expressing cells, and 20 PV-expressing cells. Cell marker quantification showed that expression of GABRA5 was detected in SLC17A7-expressing cells (Fig. 3a), SST-expressing cells (Fig. 3c1), and PV-expressing cells (Fig. 3d1) cells, but not in VIP-expressing cells
(Fig. 3b). Cells containing $\geq 10 \mathrm{mRNA}$ grains were identified as one of the four cell types (Fig. 4a). The result showed that, per $100 \mu \mathrm{m}^{3}$, the proportions of SLC17A7expressing cells and GABAergic neurons were 71.9 and $28.1 \%$, respectively (Fig. 4b). Among GABAergic neurons, the proportions of VIP-, SST-, and PV-expressing cells were $22.8,42.1$, and $35.1 \%$, respectively. GABRA5 expression in each cell type was then quantified (Fig. 4c), indicating that in SLC17A7-, VIP-, SST-, and PV-expressing cells, the percentages of cells also expressing GABRA5 were $39.7,0,8.3$, and $20 \%$, respectively. 


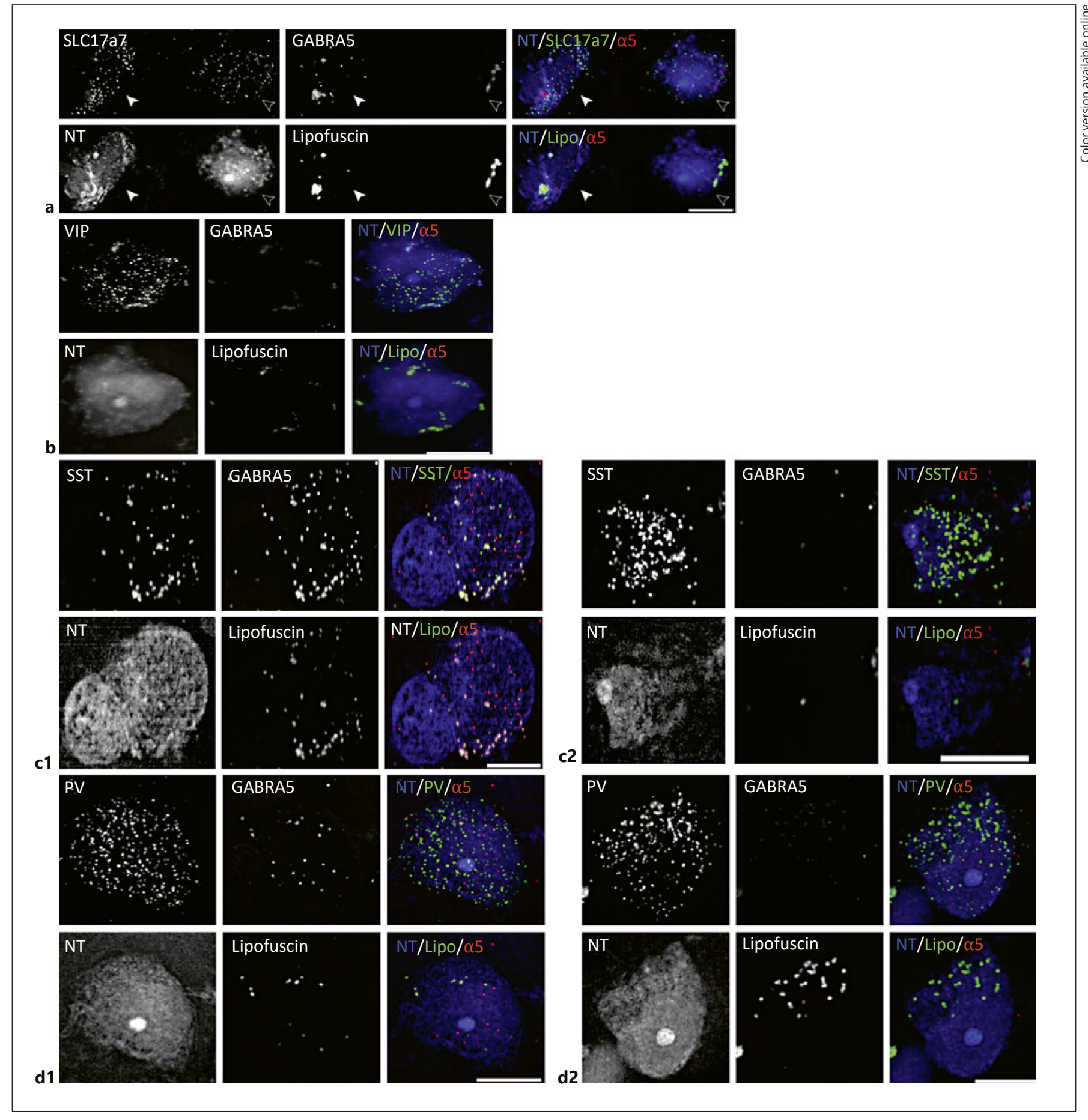

Fig. 3. GABRA5 expression in SLC17A7-, VIP-, SST-, and PV-expressing cells in human PFC. Projection images (three z-planes separated by $0.25 \mu \mathrm{m}$ ) of a human PFC tissue section, counterstained with NeuroTrace blue, and labeled for SLC17A7 with and without GABRA5 (a), VIP without GABRA5 (b), SST with (c1) and without (c2) $G A B R A 5$, and $P V$ with (d1) and without (d2) GABRA5. Solid arrowheads depict GABRA5-expressing neurons. Scale bars, $10 \mu \mathrm{m}$. 


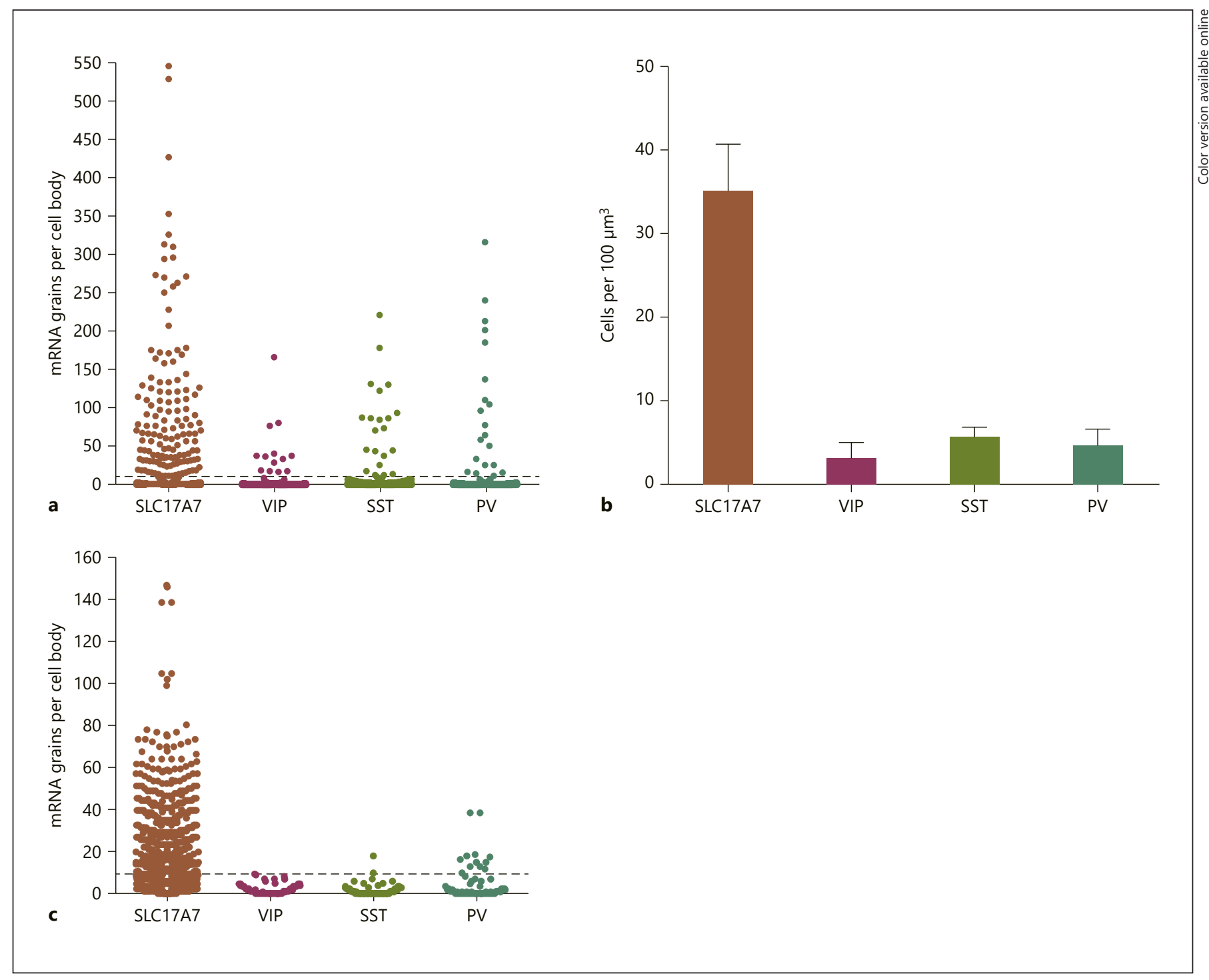

Fig. 4. Quantification of GABRA5 mRNA grains per cell body across cell types in human PFC. Cells above the cutoff ( $\geq 10$ mRNA grains) are defined as positive cell marker-expressing cells. a Cell marker mRNA grains per cell body distinguish different cell types. b Cell density of different cell types per $100 \mu \mathrm{m}^{3}$ human PFC. c GABRA5 mRNA grains per cell body expressed in SLC17A7-, VIP-, SST-, or PV-expressing cells.

\section{Discussion}

In the present study, we characterized the cellular distribution of GABRA5/Gabra5 gene expression using multiplex FISH in glutamatergic PYCs and three major groups of GABAergic neurons (PV-, VIP-, and SST-expressing neurons) in the mouse and human PFC. Our results confirm that the GABRA5/Gabra5 genes are predominantly expressed in pyramidal neurons across species, with $54 \%$ of mouse and $39.7 \%$ of human PYCs expressing these transcripts. We found an absence of GABRA5/Gabra5 expression in mouse and human VIP cells, and lower percentages of Gabra5 expression in mouse (16\%) and GABRA5 in human (20\%) PV cells. The only cross-species difference was found in SST cells: wherein Gabra5 was absent in mouse SST cells, a small proportion of human SST cells expressed GABRA5 (8\%). Given that recent studies investigating the therapeutic potential of modulating $\alpha 5-\mathrm{GABA}_{\mathrm{A}}$ Rs have shown promise in preclinical models of mood disorders [18, 27-30], 


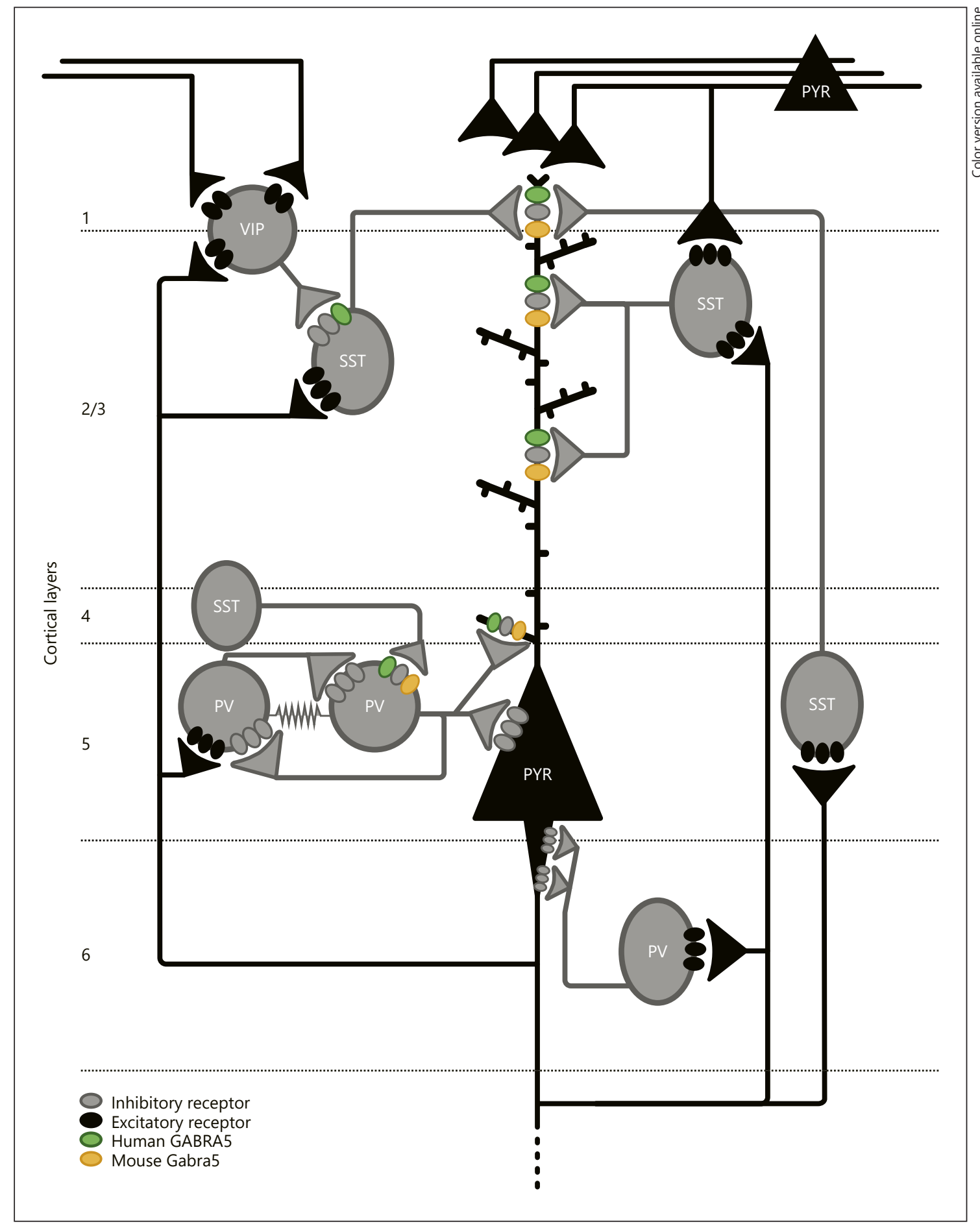

Fig. 5. Possible inhibitory roles for PFC a5-GABA ${ }_{A}$ Rs informed by human and mouse gene expression. Positive expression of the a5$\mathrm{GABA}_{\mathrm{A}} \mathrm{R}$ gene in human-only (GABRA5, green) or human and mouse (GABRA5/Gabra5, orange) PFC alongside other inhibitory GABAergic receptors (gray) targeted by SST, PV, and VIP neurons, and excitatory glutamatergic receptors (black) targeted by pyramidal neurons (PYR). Previous findings suggest that PYR a5$\mathrm{GABA}_{\mathrm{A}}$ Rs are mainly localized to dendrites $[17,34]$, whereas less is known about connections between inhibitory neurons. Across both species, $\mathrm{PFC}$ a5-GABA $\mathrm{A}_{\mathrm{A}} \mathrm{R}$ expression is predominant in PYRs (54\% mouse, $40 \%$ human) that are targeted by SST and PV neurons (among others), and moderate in PV neurons (16\% mouse, $20 \%$ human) that receive local SST and reciprocal PV connections $[8,36]$. PFC SST a5-GABA $A_{A}$ R expression was detected only in human subjects, albeit at low levels (8\%), suggesting potential VIP regulation via this mechanism [23]. 
our findings may be useful for understanding the potential influence of a5-GABA ${ }_{A} R$-selective pharmacological agents on cortical microcircuit activity at the cellular and network levels.

We first performed a descriptive analysis of cell density in mouse and human PFC. Previous studies indicate that the proportion of excitatory to inhibitory neuron populations differs between mice and humans. Specifically, the proportion of GABAergic neurons to total cells in the neocortex of rodents $(\sim 15 \%)$ is lower than that of primates (20-30\%) [3-6]. Our findings are mostly consistent with these studies, showing proportions of PYCs to GABAergic neurons at 88 vs. $12 \%$ in mice and 72 vs. $28 \%$ in humans. The RNA FISH approach employed in this study was further validated by finding high GABRA5/Gabra5 gene expression in human and mouse PYCs (human: $40 \%$; mouse: $54 \%$ ), which is consistent with previous electrophysiology and immunocytochemistry studies showing enriched $\alpha 5-G_{A B A} R$ expression localized to PYC dendrites $[17,31]$. The minor differences between our results and those of previous studies are not surprising since cortical neuron density varies based on sampled brain region and seems to vary across species [32].

SST cells are powerful regulators of the cortical microcircuit; they exhibit strong connection potential with PYCs [16] and other GABAergic neurons, including through afferents onto PV neurons [33] and reciprocal connections with VIP neurons [22]. That we found a lack of Gabra5 expression in mouse SST cells is not unexpected, since (1) a5-GABA $\mathrm{A}_{\mathrm{A}}$ localized to the apical dendrites of PYCs primarily receive GABAergic inputs from SST-expressing Martinotticells, which have a $5-G_{A B A}$ Rs as their major target, and (2) a major difference between SST and other GABAergic neuron subtypes is that SST cells strongly inhibit other neurons, but avoid targeting each other $[16,17,22]$. Taken together, these findings imply that $\alpha 5-\mathrm{GABA}_{\mathrm{A}}$ Rs are mainly targeted by presynaptic SST afferents and are not involved in inhibitory regulation of SST cells by the other GABAergic populations examined, i.e., PV or VIP neurons. Conversely, we found evidence for GABRA5 expression in human PFC SST cells. Although these represented a small proportion of total SST cells (8\%), fluorescence signals were manually inspected and considered to be true. There may be several explanations for this result. In mice, VIP-mediated inhibition of SST cells has been demonstrated through optogenetic methods [22], yet the cellular mechanisms of this inhibitory control are currently unknown. Although our data do not support the regulation of SST cells by a 5-
$\mathrm{GABA}_{\mathrm{A}}$ Rs in the mouse PFC, it is possible that such a mechanism is present in humans. Further studies are needed to assess this possibility. GABRA5/Gabra5 expression in SST cells would also suggest a tonic inhibitory mechanism to regulate SST cell activity. A distinct property of SST neurons is their high basal firing rate that persists even without excitatory input [34]. It would be expected that SST cell activity is tightly regulated to synchronize firing with other cortical neurons. Owing to the dense axonal arborization of SST cells that ascend towards, and innervate, nearby neurons across the cortical column [34], this may represent an important target for extracellular GABA that activates extrasynaptic $\mathrm{GABA}_{\mathrm{A}}$ Rs. One study found neuronal nitric oxide-expressing SST cells localized to deep cortical layers distinguished by coexpression of the $\gamma 1$ subunit, which is thought to bias $\mathrm{GABA}_{\mathrm{A}} \mathrm{Rs}$ to extrasynaptic localization [35], suggesting that these $\mathrm{GABA}_{\mathrm{A}}$ Rs may play an important role in regulating SST cell activity. Although another study revealed a5-GABA ${ }_{A}$ Rs present at synaptic sites [36], they are largely localized to extrasynaptic sites where they mediate tonic inhibition [37]. Altogether, the present findings raise the possibility of an inhibitory mechanism by which a5-GABA ${ }_{A}$ Rs may regulate the firing of a small proportion of human SST cells. However, it is not currently known whether these cells represent a unique subtype of SST cells.

Our findings showed that mouse (16\%) and human (20\%) PV cells in the PFC express moderate levels of GABRA5/Gabra5. This suggests potential innervation of PV cells by other GABAergic neurons, likely SST cells based on laminar codistribution. This hypothesis is supported by a previous study finding SST cells in layer IV of the somatosensory cortex targeting mainly fast-spiking GABAergic neurons, which largely consist of PVexpressing basket cells [33]. The majority of layer IV SST cells are X94 cells, which, unlike the long-range ascending projections of Martinotti cells, have axonal projections that profusely innervate local targets [33]. Therefore, based on anatomical proximity, PV cells are indeed likely to be innervated by local SST cells in deep cortical layers. Since $a 5-\mathrm{GABA}_{\mathrm{A}}$ Rs are a main mediator of SST cell inhibition, this further implies the possibility of SSTPV afferents. Interestingly, PV cells mainly target the soma and proximal dendrites of PYCs [21], whereas SST cells preferentially target the apical dendrites of PYCs [16]. As a result, SST-PV regulation could be an important disinhibitory mechanism for tuning the excitability of PYCs by shifting inhibition to the dendritic compartment. This could also be a cell-specific difference. PV 
neurons are mainly composed of two types of cells - basket cells and chandelier cells. A striking difference between them is that the former not only target PYCs, but also engage in PV-PV interactions, which are among the fastest signal transmissions and mediated by a1$\mathrm{GABA}_{\mathrm{A}} \mathrm{Rs}[21,38,39]$. PV cells also receive innervations from other GABAergic neurons through slower kinetic receptors that may be activated by extrasynaptic GABA from perisomatic synapses [35]. $\alpha 5-\mathrm{GABA}_{\mathrm{A}} \mathrm{Rs}$ are mostly localized to extrasynaptic sites where they mediate tonic inhibition under elevated extracellular GABA concentrations [37]. Therefore, PV neurons expressing GABRA5/Gabra5 may largely reflect chandelier cell populations rather than fast-spiking basket cells. Future studies would benefit from combining neurochemical and morphological approaches to further characterize these differences.

Our findings showed an absence of GABRA5/Gabra5 expression in human and mouse VIP cells. One explanation for this result is that, since VIP cells are uniformly modulated by cholinergic and serotonergic transmissions via ionotropic receptors [40], VIP cell activation may be more likely to depend on cholinergic rather than GABAergic transmission. This was also demonstrated in an experiment showing that blockade of acetylcholine receptors attenuated VIP cell firing rate increases, whereas no such effect was found for $\mathrm{GABA}_{\mathrm{A}} \mathrm{R}$ blockade [41]. Taken together, these studies suggest that neuromodulators such as acetylcholine, serotonin, and nicotinic agonists have crucial roles in driving the depolarization and recruitment of VIP cells and that the mechanisms underlying VIP cell regulation through GABAergic inhibition remain poorly understood. VIP cells have an essential role in regulating cortical microcircuit activity. They indirectly disinhibit PYCs and PV cells through VIP-SST cell inhibition leading to either increased (i.e., by PYC disinhibition) or decreased PYC firing (i.e., through indirectly enhancing perisomatic inhibition via $\mathrm{PV}$ cell disinhibition). Therefore, follow-up studies on the GABAergic control of VIP cells are justified to better decipher these cellular mechanisms.

The second aim of this study was to compare $G A B$ $R A 5 / G a b r a 5$ expression patterns between human and mouse PFC in order to advance our understanding of the cortical microcircuit and assess the theoretical validity of antidepressants with novel mechanisms of action. In order to remediate or bypass SST cell deficits observed in MDD subjects, interventions that potentiate SST cell input onto target receptors represent a novel therapeutic strategy. This can be achieved by enhancing GABAergic

Cell Type-Specific Gene Expression of a5-GABA $\mathrm{A}$ Rs signaling through a5-GABA ${ }_{A}$ Rs. Indeed, selectively modulating $\alpha 5-\mathrm{GABA}_{\mathrm{A}} \mathrm{Rs}$ with $\alpha 5$-selective PAM and NAM compounds induces antidepressant-like effects in rodents $[18,28]$. Although the reason for converging effects from opposite modulatory mechanisms is not yet understood, it has been suggested that SST cell regulation of cortical microcircuit activity by a $5-\mathrm{GABA}_{\mathrm{A}} \mathrm{R}$ potentiation follows an inverted $U$ curve to promote therapeutic efficacy, or alternatively that short-term inhibition leads to recurrent potentiation through cortical feedback loops, similar to recent findings on the mechanisms of rapid-acting antidepressants [19]. In the context of differential GABRA5/Gabra5 gene expression patterns across GABAergic neurons and potential species differences (Fig. 5), our study advances the potential to predict with greater accuracy how cortical microcircuit activity would be altered by administration of a5-preferring therapeutic agents. For example, our findings suggest that a5-PAM would mainly potentiate GABAergic inhibition of PYCs in the PFC, but that its potency would differ between mice and humans. Potential GABRA5 expression in human SST cells would suggest reduced SST cell firing upon a5-PAM treatment, in turn disinhibiting PYCs and PV cells, whereas this effect would not be expected in mice. Additionally, a higher proportion of human PV cells express GABRA5, suggesting a higher possibility of PV cell inhibition compared to mice, which would contribute to stronger PYC disinhibition. Therefore, a5-PAM may be more potent in targeted drug effects (i.e., enhancing PYC inhibition) in mice than in humans. However, these predictions rely on the assumption that humans and mice have similar electrophysiological properties. A more precise model of how a5-selective therapeutic agents may modulate neuronal networks would further require taking into account species differences in synaptic morphology and neurophysiological properties.

In summary, our study provides a comprehensive characterization of GABRA5/Gabra5 gene expression in PYCs and three major groups of GABAergic neurons in the human and mouse PFC. In addition to presence of a5-GABA ${ }_{\mathrm{A}} \mathrm{R}$ predominantly in PYCs, we can predict two circuit mechanisms, including $\alpha 5-G_{A B A} R$-mediated inhibition of $\mathrm{PV}$ cells across species and a unique role for a5-GABA ${ }_{\mathrm{A}}$ Rs in human SST cells, potentially indicating regulation of tonic inhibition. Our study compares gene expression patterns between humans and mice, yet future studies are warranted to investigate the underlying mechanisms that mediate the interactions between these neurons in humans. Furthermore, future studies should in- 
clude an analysis of female subjects to examine sex differences in cell-specific GABRA5/Gabra5 gene expression. Future studies would also benefit from a greater sample size and controls for the effects of RNA integrity in postmortem samples when comparing across different age and disease states. Lastly, it is important to mention that mRNA transcript levels may not be wholly reflective of cellular function. Future investigations would benefit from complementing the current experimental methods by assessing other features of cellular function, e.g., through electrophysiological techniques.

\section{Statement of Ethics}

The animal experiments conformed to internationally accepted standards and were approved by the appropriate institutional review body. Animal testing was conducted in accordance with the Canadian or US institutional animal care committee.

\section{Disclosure Statement}

E. Sibille is a coinventor on a US provisional patent application that covers the described ligands modulating the function of GABA neurons. The other authors report no biomedical financial interests or potential conflicts of interest.

\section{Funding Sources}

This work was supported by the following grants to E. Sibille: National Alliance for Research on Schizophrenia and Depression (NARSAD) award (\#25637) and a funding from the Campbell Family Mental Health Research Institute (Campbell Foundation).

\section{Author Contributions}

X. Hu, B.R. Rocco, and E. Sibille designed the study. X. Hu performed all experiments under supervision of B.R. Rocco, who developed the FISH protocol. C. Fee contributed to data analysis and figure creation. X. Hu and C. Fee wrote the manuscript. B.R. Rocco and E. Sibille edited the manuscript.

\section{References}

1 Fino E, Packer AM, Yuste R. The logic of inhibitory connectivity in the neocortex. Neuroscientist. 2013 Jun;19(3):228-37.

2 Luscher B, Fuchs T. GABAergic control of depression-related brain states. Adv Pharmacol. 2015;73:97-144.

3 Beaulieu C. Numerical data on neocortical neurons in adult rat, with special reference to the GABA population. Brain Res. 1993 Apr; 609(1-2):284-92.

4 Hendry SH, Schwark HD, Jones EG, Yan J. Numbers and proportions of GABA-immunoreactive neurons in different areas of monkey cerebral cortex. J Neurosci. 1987 May; 7(5):1503-19.

5 Gabbott PL, Bacon SJ. Local circuit neurons in the medial prefrontal cortex (areas 24a,b,c, 25 and 32) in the monkey: II. Quantitative areal and laminar distributions. J Comp Neurol. 1996 Jan;364(4):609-36.

6 Gabbott PL, Dickie BG, Vaid RR, Headlam AJ, Bacon SJ. Local-circuit neurones in the medial prefrontal cortex (areas 25, 32 and $24 \mathrm{~b})$ in the rat: morphology and quantitative distribution. J Comp Neurol. 1997 Jan;377(4): 465-99.

7 Markram H, Toledo-Rodriguez M, Wang Y, Gupta A, Silberberg G, Wu C. Interneurons of the neocortical inhibitory system. Nat Rev Neurosci. 2004 Oct;5(10):793-807.

8 Rudy B, Fishell G, Lee S, Hjerling-Leffler J. Three groups of interneurons account for nearly $100 \%$ of neocortical GABAergic neurons. Dev Neurobiol. 2011 Jan;71(1):45-61.
9 Condé F, Lund JS, Jacobowitz DM, Baimbridge KG, Lewis DA. Local circuit neurons immunoreactive for calretinin, calbindin D$28 \mathrm{k}$ or parvalbumin in monkey prefrontal cortex: distribution and morphology. J Comp Neurol. 1994 Mar;341(1):95-116.

10 Barinka F, Druga R. Calretinin expression in the mammalian neocortex: a review. Physiol Res. 2010;59(5):665-77.

11 Sibille E, Morris HM, Kota RS, Lewis DA. GABA-related transcripts in the dorsolateral prefrontal cortex in mood disorders. Int $\mathrm{J} \mathrm{Neu}$ ropsychopharmacol. 2011 Jul;14(6):721-34.

12 Tripp A, Kota RS, Lewis DA, Sibille E. Reduced somatostatin in subgenual anterior cingulate cortex in major depression. Neurobiol Dis. 2011 Apr;42(1):116-24.

13 Guilloux JP, Douillard-Guilloux G, Kota R, Wang X, Gardier AM, Martinowich K, et al. Molecular evidence for BDNF- and GABArelated dysfunctions in the amygdala of female subjects with major depression. Mol Psychiatry. 2012 Nov;17(11):1130-42.

14 Douillard-Guilloux G, Lewis D, Seney ML, Sibille E. Decrease in somatostatin-positive cell density in the amygdala of females with major depression. Depress Anxiety. 2017 Jan;34(1): 68-78.

15 Seney ML, Tripp A, McCune S, Lewis DA, Sibille E. Laminar and cellular analyses of reduced somatostatin gene expression in the subgenual anterior cingulate cortex in major depression. Neurobiol Dis. 2015 Jan;73:2139.
16 Silberberg G, Markram H. Disynaptic inhibition between neocortical pyramidal cells mediated by Martinotti cells. Neuron. 2007 Mar; 53(5):735-46.

17 Ali AB, Thomson AM. Synaptic alpha 5 subunit-containing GABAA receptors mediate IPSPs elicited by dendrite-preferring cells in rat neocortex. Cereb Cortex. 2008 Jun;18(6): 1260-71.

18 Piantadosi SC, French BJ, Poe MM, Timić T, Marković BD, Pabba M, et al. Sex-Dependent Anti-Stress Effect of an a5 Subunit Containing GABAA Receptor Positive Allosteric Modulator. Front Pharmacol. 2016 Nov;7: 446

19 Fee C, Banasr M, Sibille E. Somatostatin-Positive Gamma-Aminobutyric Acid Interneuron Deficits in Depression: Cortical Microcircuit and Therapeutic Perspectives. Biol Psychiatry. 2017 Oct; 82(8):549-59.

20 Ferando I, Mody I. Interneuronal GABAA receptors inside and outside of synapses. Curr Opin Neurobiol. 2014 Jun;26:57-63.

21 Nusser Z, Sieghart W, Benke D, Fritschy JM, Somogyi P. Differential synaptic localization of two major gamma-aminobutyric acid type A receptor alpha subunits on hippocampal pyramidal cells. Proc Natl Acad Sci USA. 1996 Oct;93(21):11939-44.

22 Pfeffer CK, Xue M, He M, Huang ZJ, Scanziani M. Inhibition of inhibition in visual cortex: the logic of connections between molecularly distinct interneurons. Nat Neurosci. 2013 Aug; 16(8):1068-76. 
23 Caputi A, Rozov A, Blatow M, Monyer H. Two calretinin-positive GABAergic cell types in layer $2 / 3$ of the mouse neocortex provide different forms of inhibition. Cereb Cortex. 2009 Jun; 19(6):1345-59.

24 Jiang X, Shen S, Cadwell CR, Berens P, Sinz F, Ecker AS, et al. Principles of connectivity among morphologically defined cell types in adult neocortex. Science. 2015 Nov; 350 (6264):aac9462.

25 Wang F, Flanagan J, Su N, Wang LC, Bui S, Nielson A, et al. RNAscope: a novel in situ RNA analysis platform for formalin-fixed, paraffin-embedded tissues. J Mol Diagn. 2012 Jan;14(1):22-9.

26 Rocco BR, Oh H, Shukla R, Mechawar N, Sibille E. Fluorescence-based cell-specific detection for laser-capture microdissection in human brain. Sci Rep. 2017 Oct;7(1):14213.

27 Koh MT, Rosenzweig-Lipson S, Gallagher M. Selective GABA(A) a5 positive allosteric modulators improve cognitive function in aged rats with memory impairment. Neuropharmacology. 2013 Jan;64:145-52.

28 Fischell J, Van Dyke AM, Kvarta MD, LeGates TA, Thompson SM. Rapid Antidepressant Action and Restoration of Excitatory Synaptic Strength After Chronic Stress by Negative Modulators of Alpha5-Containing GABAA Receptors. Neuropsychopharmacology. 2015 Oct;40(11):2499-509.
29 Zanos P, Nelson ME, Highland JN, Krimmel SR, Georgiou P, Gould TD, et al. A negative allosteric modulator for a5 subunit-containing GABA receptors exerts a rapid and persistent antidepressant-like action without the side effects of the NMDA receptor antagonist ketamine in mice. eNeuro 2017 Mar;4(1): ENEURO.0285-16.2017.

30 Xu NZ, Ernst M, Treven M, Cerne R, Wakulchik M, Li X, et al. Negative allosteric modulation of alpha 5-containing GABAA receptors engenders antidepressant-like effects and selectively prevents age-associated hyperactivity in tau-depositing mice. Psychopharmacology (Berl). 2018 Apr;235(4):1151-61.

31 Sperk G, Schwarzer C, Tsunashima K, Fuchs $\mathrm{K}$, Sieghart W. GABA(A) receptor subunits in the rat hippocampus I: immunocytochemical distribution of 13 subunits. Neuroscience. 1997 Oct;80(4):987-1000.

32 Collins CE, Airey DC, Young NA, Leitch DB, Kaas JH. Neuron densities vary across and within cortical areas in primates. Proc Natl Acad Sci USA. 2010 Sep;107(36):15927-32.

$33 \mathrm{Xu} \mathrm{H}$, Jeong HY, Tremblay R, Rudy B. Neocortical somatostatin-expressing GABAergic interneurons disinhibit the thalamorecipient layer 4. Neuron. 2013 Jan;77(1):155-67.

34 Urban-Ciecko J, Barth AL. Somatostatin-expressing neurons in cortical networks. Nat Rev Neurosci. 2016 Jul;17(7):401-9.

35 Paul A, Crow M, Raudales R, He M, Gillis J, Huang ZJ: Transcriptional architecture of synaptic communication delineates GABAergic neuron identity. Cell. 2017 Oct;171(3): 522-539.e520.
36 Serwanski DR, Miralles CP, Christie SB, Mehta AK, Li X, De Blas AL. Synaptic and nonsynaptic localization of GABAA receptors containing the alpha5 subunit in the rat brain. J Comp Neurol. 2006 Nov;499(3):458-70.

37 Caraiscos VB, Elliott EM, You-Ten KE, Cheng VY, Belelli D, Newell JG, et al. Tonic inhibition in mouse hippocampal CA1 pyramidal neurons is mediated by alpha5 subunitcontaining gamma-aminobutyric acid type $\mathrm{A}$ receptors. Proc Natl Acad Sci USA. 2004 Mar; 101(10):3662-7.

38 Galarreta M, Hestrin S. Electrical and chemical synapses among parvalbumin fast-spiking GABAergic interneurons in adult mouse neocortex. Proc Natl Acad Sci USA. 2002 Sep; 99(19):12438-43.

39 Farrant M, Nusser Z. Variations on an inhibitory theme: phasic and tonic activation of GABA(A) receptors. Nat Rev Neurosci. 2005 Mar;6(3):215-29.

40 Férézou I, Cauli B, Hill EL, Rossier J, Hamel E, Lambolez B. 5-HT3 receptors mediate serotonergic fast synaptic excitation of neocortical vasoactive intestinal peptide/cholecystokinin interneurons. J Neurosci. 2002 Sep; 22(17):7389-97.

41 Karnani MM, Jackson J, Ayzenshtat I, Tucciarone J, Manoocheri K, Snider WG, et al. Cooperative Subnetworks of Molecularly Similar Interneurons in Mouse Neocortex. Neuron. 2016 Apr;90(1):86-100. 